\title{
La sociologie et la géographie : concepts, analogies, métaphores
}

Jacques Coenen-Huther

\section{(2) OpenEdition}

\section{Journals}

Édition électronique

URL : http://journals.openedition.org/ress/718

DOI : $10.4000 /$ ress. 718

ISSN : 1663-4446

Éditeur

Librairie Droz

\section{Édition imprimée}

Date de publication : 1 février 2000

Pagination : 141-158

ISBN : 2-600-00409-2

ISSN : 0048-8046

\section{Référence électronique}

Jacques Coenen-Huther, "La sociologie et la géographie : concepts, analogies, métaphores », Revue européenne des sciences sociales [En ligne], XXXVIII-117| 2000, mis en ligne le 17 décembre 2009, consulté le 19 avril 2019. URL : http://journals.openedition.org/ress/718 ; DOI : 10.4000/ress.718 


\section{LA SOCIOLOGIE ET LA GÉOGRAPHIE: CONCEPTS, ANALOGIES, MÉTAPHORES}

Des générations d'étudiants en sociologie ont été vigoureusement mis en garde contre un déterminisme géographique qui ne pourrait que nuire au développement de la sociologie comme projet scientifique propre. «Depuis cinquante ans, tout ce bruit s'est apaisé », notait Maximilien Sorre à la fin des années cinquante (1957, p. 7). A l'époque, ce bel optimisme était certainement prématuré. Actuellement, les polémiques se sont peut-être tues mais la méfiance n'a pas disparu pour autant. Cette méfiance longuement entretenue est sans nul doute la conséquence des débats sur le «facteur prédominant» qui ont marqué les débuts de la sociologie au $\mathrm{XIX}^{\mathrm{e}}$ siècle. Au milieu du $\mathrm{XX}^{\mathrm{e}}$ siècle, le combat contre la monocausalité ne semblait pas encore complètement gagné par la sociologie moderne, d'autant moins qu'une vulgate marxiste largement répandue tendait sans cesse à la faire ressurgir. Il semblait donc toujours impérieux de se prémunir contre les dangers d'un «monisme géographique» qui semblait ne pouvoir favoriser qu'une sociologie de pacotille. Quelques décennies plus tard, on est frappé par tout ce que cette attitude paraît avoir comporté de malentendus. Dans un ouvrage d'histoire de la sociologie qui fit longtemps autorité ${ }^{1}$, Pitirim Sorokin ${ }^{2}$ consacre un chapitre à «l'école géographique» où sont cités comme participant d'un projet commun - à savoir «insister sur divers effets des conditions géographiques sur la conduite et la psychologie de l'homme, et sur l'organisation sociale, les processus sociaux, et les destinées historiques du groupe » (1928, 1938, p. 93) des hommes aussi différents que Lamarck, Humboldt, Le Play, Ratzel, Elisée Reclus, Vidal de la Blache, Brunhes, Vallaux et Huntington entre autres (ibid.). Au début des années soixante encore, Georges Gurvitch reprend la même expression «l'école géographique en sociologie» - pour qualifier une de ces «soi-disant écoles sociologiques » qui ont «cherché à décomposer la réalité sociale en facteurs isolés » et qui ont «ainsi laissé échapper le social proprement dit» (1963, pp. 5051). Pour Gurvitch, il est vrai, Ratzel, Brunhes et Le Play sont les promoteurs de ce projet visant à «expliquer la réalité sociale à partir de phénomènes naturels ${ }^{3}$

\footnotetext{
Selon les termes de Don Martindale, «perhaps the finest single systematic study of sociological theory that America has produced» (1981, p. 123).

2 Sociologue russe, devenu américain à la suite de son bannissement de l'URSS (voir à ce sujet l'article de Lenine dans la Pravda du 21 novembre 1918: «Les précieux aveux de Pitirim Sorokin », Euvres choisies, Editions du Progrès, Moscou, 1962, pp. 61-70).

3 La remarque est particulièrement injuste en ce qui concerne Le Play qui a plutôt joué un rôle de médiateur entre la sociologie et la géographie comme l'ont bien montré Jean-Michel Berthelot (1991, pp. 13-16) et Bernard Valade (1996, p. 341).
} 
tandis que Vidal de la Blache, Maximilien Sorre et quelques autres ont «rendu cette position impossible» (ibid., p. 51). Même aux Pays-Bas, où la sociologie est née de la géographie ${ }^{4}$, son émancipation institutionnelle - partant de la géographie humaine ou sociale (sociale geografie) enseignée spécialement à Utrecht en passant par la «sociographie» de l'Ecole d'Amsterdam - est généralement présentée comme la constitution progressive d'une véritable science du social où «l'aspect géographique, à savoir la détermination de la vie de groupe par le milieu physique... passe progressivement à l'arrière-plan »(Van Doorn, 1964, pp. 35-41). Et quand est souligné le caractère «pragmatique et empirique $»^{5}$ de la sociologie néerlandaise, c'est généralement pour l'attribuer aux origines géographiques de la discipline (Vervoort, 1979, p. 105).

Bien sûr, les sociologues de la première moitié du $\mathrm{XX}^{\mathrm{e}}$ siècle restaient confrontés à des conceptions «d'une naïveté romantique», mettant l'accent sur «l'action directe des lieux sur les esprits» (Dupréel, 1948, pp. 383-384), auxquelles de nombreux géographes avaient souscrit et dont on trouve une puissante expression littéraire dans La colline inspirée de Maurice Barrès (1913). Mais peut-être eussent-ils trouvé davantage d'appuis du côté d'une géographie humaine en pleine évolution (Mucchielli, 1998, Chap. 11) s'ils les avaient cherchés plus sérieusement et s'ils n'avaient pas tenté de lui substituer une «morphologie sociale» au statut mal défini. En effet, c'est Elisée Reclus qui, au XIX ${ }^{\mathrm{e}}$ siècle déjà, déclarait que «si la géographie proprement dite, qui s'occupe seulement de la forme et du relief de la planète, nous expose l'état passif des peuples... en revanche la géographie historique et statistique nous montre les hommes entrés dans leur rôle actif et reprenant le dessus par le travail sur le milieu qui les entoure» (1875, pp. 7-8). Brunhes, pour sa part, invite clairement à ne jamais méconnaître «même en géographie, tout ce qui dérive sur la terre des décisions libres ou des actes irréfléchis des hommes » (1910, 1956, p. 278). Avec Vallaux, il cherche à établir la géographie humaine - en tant que géographie de l'histoire ou géographie politique - à l'écart du «déterminisme du milieu selon Taine et de celui des influences ethniques selon Gobineau » (Brunhes et Vallaux, 1921, p. 3). Quant à Vidal de la Blache, c'est avec autant de netteté qu'il évoque «l'entrecroisement perpétuel des faits sociaux et des faits géographiques» (1922, p. 98). Il semble donc bien que dans les milieux de sociologues, les «cousins mal-aimés » ${ }^{6}$ soient avant tout des cousins éloignés, peu connus, moins connus que les historiens avec lesquels les sociologues entretiennent également des rapports de cousinage ambivalent. Quand la qualité de leurs travaux fut reconnue, ce fut dans un rôle auxiliaire qu'on sembla vouloir les confiner. Et ceci semble avoir été bien perçu par les géographes. Vidal de la Blache note que «pour la plupart des historiens et des sociologues, la géographie n'intervient qu'à titre consultatif » (1922, p. 5) ${ }^{7}$. Pourtant, c'est bien une préoccupation de synthèse novatrice qui s'exprima

Steinmetz, généralement considéré comme le fondateur de la sociologie néerlandaise, était à la fois ethnologue et géographe (Van Doorn, 1964, pp. 36-37).

5 Lisons: peu soucieux d'élaboration théorique.

6 L'expression est de Claude Raffestin, répliquant à l'auteur de ces lignes qui avait évoqué le cousinage de la sociologie et de la géographie.

7 Moi-même, j'ai eu l'occasion de suggérer des possibilités de collaboration entre géographie et sociologie au stade de l'activité typologique, dont je reconnais volontiers après coup qu'elles ne 
régulièrement de ce côté. Brunhes cite de façon approbatrice un économiste qui envisage la géographie humaine comme «destinée à rénover toutes les théories sociologiques qui spéculent sur je ne sais quel homme abstrait ${ }^{8}$ (Brunhes, 1910, pp. 282-283). Pour Vidal de la Blache, «la géographie humaine... apporte une conception nouvelle des rapports entre la terre et l'homme, conception suggérée par une connaissance plus synthétique des lois physiques qui régissent notre sphère et des relations entre les êtres vivants qui la peuplent » (1922, p. 3; souligné par JCH). Plus tard, Pierre George affirmera que la géographie humaine «déborde la compétence des autres sciences humaines, y compris la sociologie en se définissant comme recherche de toutes les corrélations et de toutes les causalités concernant la situation actuelle et les virtualités [des diverses] collectivités » (1966, p. 4; souligné par JCH).

Dans ces conditions, à quoi tient l'incompréhension ou le dédain manifesté si fréquemment par les sociologues? Faut-il n'y voir qu'un exemple parmi d'autres de rivalité académique et, précisément, de compétition pour l'activité de synthèse théorique? Dans leur trivialité apparente, ces éléments jouent très probablement un rôle. Mais les obstacles aux échanges intellectuels se situent aussi sur un autre plan. Pour se constituer en discipline propre et affirmer la spécificité de son objet, la sociologie dut se donner des règles de méthode qui l'affranchissent de toute sujétion aux sciences de la nature. Cette préoccupation majeure se manifesta soit dans l'injonction durkheimienne d'expliquer «le social avec du social» soit dans la priorité conférée par l'orientation weberienne à la recherche du sens des actions par voie de compréhension (Verstehen). Qu'on se place donc dans une perspective épistémologique continuiste - adoptant les mêmes règles fondamentales pour les sciences humaines et pour les sciences de la nature - ou dans une perspective discontinuiste - insistant sur la spécificité irréductible des sciences humaines - le souci récurrent des sociologues est de définir leur raison d'être - le social comme réalité sui generis - exclusivement en termes de rapports d'interdépendance et de complémentarité entre humains. Dès lors, toute autre variable apparaît comme parasite. L'idée, par exemple, que «la nature impose à nos activités des conditions restrictives» (Brunhes, 1910, p. 268) n'est acceptée qu'avec réserves. Quant à la notion de «l'homme comme facteur géographique», à la fois «actif et passif» dans un environnement naturel qui puisse être autre chose que «la scène» sur laquelle se déroule son activité (Vidal de la Blache, 1922, pp. 5-13), elle est troublante dans la mesure où elle ne peut qu'imposer de longs détours - c'est-à-dire une succession de variables intermédiaires - entre l'être humain et son semblable.

La géographie humaine au contraire se situe à l'intersection des sciences de la nature et des sciences sociales. Son projet scientifique ne peut que l'inciter à franchir constamment cette barrière interdisciplinaire. Ceci n'est pas sans influence

rendaient pas justice à tout le potentiel de la géographie humaine (Coenen-Huther, 1989, p. 11). Il est vrai que je m'appuyais sur une proposition singulièrement modeste de Pierre George, assignant à la géographie le soin «de dresser la répartition des types élaborés par la typologie sociale» (George, 1966, p. 16; souligné par JCH).

8 à savoir, isolé de son contexte d'activité. La citation est de Georges Gariel, dans la Revue d'économie politique (1903, pp. 802-826). Cet auteur ne fait que remettre en question les prétentions de validité universelle de certaines théories sociologiques et ne susciterait actuellement plus la moindre réticence chez les sociologues. 
sur les modes de raisonnement et les procédés d'argumentation des géographes. Dans un article consacré aux mouvements migratoires ${ }^{9}$, Claude Raffestin, évoquant la notion de «besoin», s'applique à en faire ressortir la complexité (1992, pp. 695-696). Cette démarche intellectuelle ne peut que recueillir l'assentiment de tous les sociologues modernes qui ont depuis longtemps cessé de voir dans les besoins humains un invariant anthropologique et qui en reconnaissent volontiers le caractère socialement et historiquement déterminé. Mais Raffestin, citant Laborit, présente le besoin comme «la quantité d'énergie et d'information nécessaires au maintien d'une structure vivante » (ibid., p. 696). Il observe que si l'on peut calculer assez aisément le nombre de calories nécessaires à un être humain, il n'en va pas de même de «la quantité d'information» requise. Ceci l'amène à souligner la nécessité de prendre en compte non seulement «des motivations physiologiques mais encore des motivations psycho-sensorielles et des motivations symboliques ou socio-culturelles ». Il en vient ainsi à rappeler qu'on ne peut nourrir une population «avec des produits qui présentent pour elle des connotations négatives du point de vue symbolique» (ibid.). Cette conclusion, incontestablement, rejoint à nouveau celles de tous les sociologues qui se sont intéressés à la question ${ }^{10}$. En revanche, les étapes intermédiaires du raisonnement - la référence à la biologie ainsi que la mise en parallèle de la quantité d'énergie et de la quantité d'information - ne peuvent que susciter leur méfiance ${ }^{11}$. A tort ou à raison, la majorité d'entre eux y verront sans doute un retour - inutile au demeurant - à une forme de «biologisme» que Sorre lui-même qualifiait de «conception périmée de la sociologie» (1957, p. 117). Un accord sur le fond peut ainsi être masqué par le choix d'un cadre de référence qui dépasse les frontières disciplinaires habituelles. Mais quand notre auteur, poursuivant son argumentation, en vient à comparer le besoin à un «signe linguistique» dont «la face signifiante serait celle de l'énergie et la face signifiée celle de l'information» (Raffestin, 1992, p. 696), il se réhabilite quelque peu aux yeux de ses collègues sociologues dans la mesure où il emprunte le vocabulaire de la linguistique, discipline qui, pour nombre d'entre eux, a succédé à la physique et à la biologie comme «science-modèle » (Coenen-Huther, 1989, p. 3).

Quoi qu'il en soit, la géographie, avec sa prédisposition intrinsèque à l'interdisciplinarité, se trouve aux prises, beaucoup plus souvent que la sociologie, avec deux catégories de structures causales. Tout d'abord, des structures causales complexes où «plusieurs variables explicatives sont elles-mêmes dépendantes d'autres variables explicatives » (Boudon, 1967, p. 36) et qui sont typiques des processus étudiés par les sciences sociales; le modèle de causalité correspondant à l'analyse durkheimienne de la relation entre le suicide et les facteurs cosmiques en fournit un bon exemple (ibid.). Ensuite, des structures causales simples, c'est-

9 Article que l'auteur de ces lignes a eu le privilège de pouvoir publier dans la «Revue Suisse de Sociologie» dont il était alors le directeur, après l'avoir sollicité au grand dam de certains de ses collègues sociologues.

10 A ce sujet, voir par exemple, Mendras et Forsé (1983, Chap. 3 «La diffusion des innovations », pp. 73-103).

11 Bien sûr, la communauté scientifique des géographes n'est pas nécessairement plus homogène que celle des sociologues et d'autres géographes pourraient également manifester des réserves à l'égard de ce mode de présentation. 
à-dire des structures causales typiques des situations d'expérimentation sur les phénomènes naturels, où «la simple existence d'une relation statistique suffit à prouver l'existence d'une relation causale, à condition qu'elle apparaisse confirmée dans des contextes différents » (ibid., pp. 32-33); un tel schéma apparaît par exemple lorsqu'on cherche à déterminer l'influence de l'utilisation d'un engrais sur le rendement d'une terre (ibid., pp. 33-34). Le géographe n'aura vraisemblablement aucune difficulté à envisager l'existence simultanée de ces deux modèles de causalité. Le sociologue en revanche est formé à considérer avec suspicion les structures proches des situations d'expérimentation. N'oublions pas que la méthodologie sociologique s'est développée en partie contre le postulat selon lequel l'existence d'une relation statistique, si elle régulièrement confirmée, est preuve d'une relation causale ${ }^{12}$.

On doit à Eugène Dupréel, philosophe et sociologue belge de la première moitié du $\mathrm{XX}^{\mathrm{e}}$ siècle, une tentative cohérente - et assez neuve en son temps - d'intégration des raisonnements sociologiques et des modes de pensée de la géographie. Pour celui-ci, les facteurs géographiques «conditionnent la complémentarité des rapports sociaux » et exercent toujours une influence sur «la répartition de la force sociale», c'est-à-dire du pouvoir (Dupréel, 1948, p. 383). Mais ces facteurs - climat, nature du sol, obstacles naturels, etc. - n'agissent sur les activités humaines que de façon indirecte, l'état des techniques constituant à cet égard un facteur intermédiaire. Les progrès techniques modifient l'action des facteurs géographiques, «le plus souvent en atténuant considérablement leur influence» (ibid.). Ainsi, des «choses matérielles» se combinent avec des «facteurs psychologiques» dans des «rapports sociaux» et forment des «synthèses sui generis» qui constituent l'objet de la sociologie. Le rapport social est donc «une synthèse d'actes et de choses » et ceci conduit à rapprocher la sociologie générale d'une «philosophie de la technique» (ibid., p. 7) pour laquelle la technique est l'élément-charnière entre la nature et la culture. C'est ainsi la technique qui rend l'être humain «plutôt actif» ou «plutôt passif » face au milieu naturel, pour reprendre une formulation vidalienne. Ce qui fait pour l'époque l'originalité de l'analyse proposée par Dupréel, c'est que sous la rubrique «technique», il place les techniques «matérielles» mais aussi les techniques «sociales», c'est-à-dire les modes d'action sur l'organisation des collectivités humaines ${ }^{13}$. Il rejoint ainsi tous ceux géographes, historiens, sociologues - qui recherchent l'explication de la permanence ou du changement dans «l'action d'un complexe» et pour qui «telle ou telle technique ou tel groupe de techniques pris à part» ne saurait suffire à fonder

12 Raymond Boudon a bien montré que c'est la remise en question de ce postulat - tant par Tarde que par Durkheim - qui a permis de dépasser les présupposés de la criminologie positiviste italienne tels qu'ils se manifestaient dans les travaux de Lombroso (1967, pp. 32 et ss). Ultérieurement, il est vrai, Boudon a indiqué avoir perçu «le caractère plutôt heuristique qu'explicatif de l'analyse causale» (1986, note 11, p. 314). Ceci ne me paraît néanmoins pas réduire l'importance de la distinction entre modèles de causalité pour l'objet de mon propos.

13 Plus tard, feu Pierre Gourou se situera dans la même optique lorsqu'il proposera une distinction entre les techniques de production et les «techniques d'encadrement» (1973), comme le rappellent fort opportunément Denise Pumain et Jean-Bernard Racine (1999, p. 78). Voir aussi à ce sujet les réflexions de Giovanni Busino sur les droits de propriété comme «technologie» assurant la performance des rapports humains et sociaux (1986, Chap. II, p. 62). 
une explication si l'on n'y ajoutait pas «les rapports entre les hommes » (Sorre, 1957, p. 72). Chez Dupréel comme chez d'autres, le raisonnement en termes de facteurs reste cependant un obstacle conceptuel majeur à la progression d'une vision globale de l'humanité aux prises avec un environnement naturel beaucoup moins souvent isolable des influences humaines qu'on a pu l'imaginer. C'est Vidal de la Blache qui fit observer à cet égard que la période historique n'est que «le dernier acte du drame humain » et que l'homme «a influé, plus anciennement et plus universellement qu'on ne pensait, sur le monde vivant» (1922, p. 8). Dans cette perspective d'interaction constante et de longue durée, la prise en compte du «facteur géographique», du «facteur démographique», du «facteur politique», etc... ne laisse pas de favoriser en effet les perpectives diverses, partielles et concurrentes. Sur ce point Gurvitch avait certainement raison encore qu'il ne cessât point de se tromper de cible et d'adopter un ton inutilement polémique, réminiscent de ses antécédents bolchéviques ${ }^{14}$.

Du côté des sociologues, c'est dans les années soixante-dix, quand une sociologie des acteurs se substitua à une sociologie des facteurs qu'augmentèrent les chances d'une approche intégrée, rejoignant l'ambition de synthèse de la géographie humaine. Les sociologues cessèrent peu à peu de se soucier prioritairement du poids respectif de facteurs divers pour se tourner vers l'analyse de stratégies d'acteurs individuels ou collectifs. Par rapport à la période du fonctionnalisme triomphant, le changement de perspective s'affirme nettement chez des auteurs comme Crozier et Friedberg lorsque ceux-ci invitent à «tirer toutes les conséquences du caractère irréductiblement indéterminé, c'est-à-dire politique, des systèmes sociaux» (1977, p. 25; souligné par JCH). Dans une conception nouvelle des ensembles systémiques humains, les acteurs disposent, dans le cadre des contraintes imposées par le système, «d'une marge de liberté qu'ils utilisent de façon stratégique». Le pouvoir - la force sociale aurait dit Dupréel, le précurseur - devient alors «le mécanisme central et inéluctable de régulation de l'ensemble» (ibid.), vérité que les géographes ont découverte de leur côté lorsqu'ils s'interrogent sur les modes spécifiques d'aménagement de l'espace par les collectivités humaines. A peu près à la même époque mais de manière indépendante, Claude Raffestin présente le pouvoir comme «consubstantiel à toute relation» (1980, p. 2), ce que les sociologues ne peuvent qu'approuver, à condition, bien entendu, de prendre en compte la distinction utilement rappelée par François Chazel entre «conception relationnelle» et «conception substantialiste» du pouvoir (1992, p. 199). Mais ceci conduit Raffestin à affirmer que « toute géographie humaine est politique» (ibid., p. 244) et à s'interroger sur les acteurs et leurs stratégies (ibid., $4^{\mathrm{e}}$ partie, chap. II). La notion même de «stratégie» - tant pour le géographe que pour le sociologue - suggère des actions jouant sur toute une palette de ressources possibles: ressources humaines diversement agencées certes, mais aussi ressources naturelles ou encore ressources naturelles modifiées, voire modelées, par l'action humaine. Les conditions sont ainsi mieux réunies pour qu'on prenne davantage conscience de ce que Maximilien Sorre appelait la «zone basale» des deux disciplines (1957, p. 52).

14 On sait qu'à l'instar de Sorokin, Georges Gurvitch fut un révolutionnaire russe, contraint ultérieurement à l'exil. 
Explorons cette «zone basale» en gardant présente à l'esprit la distinction piagétienne entre domaine matériel et domaine conceptuel des disciplines scientifiques qui est ici tout à fait pertinente (Piaget, 1967, p. 1173). Le terme de «stratégie», qu'on l'emploie dans le sens courant ou, à la manière de Bourdieu, pour qualifier des lignes d'action dont on n'est pas nécessairement conscient ${ }^{15}$, semble bien être devenu - pour les sociologues comme pour les géographes - la traduction la plus adéquate, tenant compte de l'autonomie des acteurs et des diverses contingences, d'un souci commun de mise en évidence des interdépendances. Mais cette notion d'interdépendance, quelle en est la nature? Quels en sont les ingrédients? Pour le sociologue, il s'agit de l'interdépendance des rapports sociaux, avec une nette tendance - Vidal de la Blache avait raison sur ce point - à considérer l'environnement naturel comme une «scène» dépourvue d'existence propre, sauf dans les cas limites où les contraintes sont extrêmement fortes. L'analyse porte ainsi sur des « vecteurs de même espèce» ou, si l'on préfère, sur les «formes diverses d'une même réalité» (George, 1966, pp. 12-13). Pour le géographe en revanche, l'ambition de saisir une interdépendance significative ne peut être satisfaite qu'à condition de prendre en compte des «forces de natures différentes » obéissant tantôt à des lois physiques tantôt à des normes de comportement issues du contexte socio-culturel (ibid.). Dans les deux cas, la préoccupation de l'interdépendance se manifesta longtemps par le recours à la métaphore organiciste. Pendant tout le $\mathrm{XIX}^{\mathrm{e}}$ siècle et au-delà, le développement de la sociologie fut porté au moins en partie par diverses formes d'organicisme positiviste, puisant leur inspiration dans l'analogie entre une société organisée et un être vivant. Les termes de «grand organisme», pour désigner la société dans son ensemble, se retrouvent sous la plume d'Auguste Comte et de Durkheim. Du côté des géographes, l'usage métaphorique de la notion d'organisme ne semble pas non plus avoir été dédaigné, encore qu'il ne paraisse pas avoir eu les mêmes conséquences théoriques en géographie qu'en sociologie. Brunhes célèbre «l'idée la plus haute,... l'idée du tout terrestre,... la conception de l'unité terrestre» $(1910,1956$, p. 12). Après avoir cité longuement Claude Bernard décrivant dans son Introduction à la médecine expérimentale, les organismes complexes et la «solidarité organique ou sociale» qui les maintient en vie dans une sorte de mouvement perpétuel, il concède que l'expression d' «organisme terrestre » est peut-être trop hardie mais il tient à conserver l'idée de solidarité organique «entre tous les phénomènes de la machine terrestre» (ibid., p. 14). Pour Henri Baulig, il convient de concevoir la Terre «non comme un support inerte, mais comme un être doué d'une activité propre $»^{16}$.

Au-delà de ces tendances superficiellement semblables, peut-on désigner un esprit proprement géographique qui se distinguerait d'un esprit spécifiquement sociologique? De fait, les expressions «esprit sociologique» et «esprit géographique» ont été utilisées en tant que telles. On pourrait convenir qu'avoir l'esprit sociologique, c'est être particulièrement sensible à tout ce que l'ordre social, avec ses relations d'interdépendance et de complémentarité ainsi que les contraintes

15 Ce qui est le cas, par exemple, lorsque Bourdieu évoque la relation entre «les stratégies des familles » et la logique de l'institution scolaire (1994, pp. 39 et ss).

16 Cité par Maximilien Sorre pour illustrer «le sens des ensembles et de l'interdépendance de leurs parties » qui lui paraît caractériser la géographie (1957, p. 40). 
structurelles qui en résultent, exerce comme influences sur nos comportements, nos façons de penser, nos relations avec nos semblables ${ }^{17}$. Pour Brunhes, avoir l'esprit géographique, c'est notamment «s'efforcer de constater avec exactitude où se produit le phénomène étudié » $(1910,1956$, p. 282). Plus près de nous, Claude Raffestin présente la géographie comme une discipline «souvent rebelle à l'introduction de notions qui ne sont pas l'objet d'une traduction spatiale immédiate» (1980, p. 1). Récemment encore, un géographe américain, Robert David Sack, rappelait l'importance pour la géographie des notions de «place» et «space» tout en insistant sur le fait que ces deux concepts «substantiate the long-standing claim that, at the academic level, geography integrates and synthetizes by interconnecting concepts that other fields take apart» $(1997$, p. 1). D'un point de vue sociologique, s'il fallait ramener tout ceci à une formule lapidaire, on serait tenté de dire que la sociologie se préoccupe avant tout du lien social alors que la géographie humaine y joint un intérêt plus précis pour le lieu où le social se manifeste et exerce ses effets. Sans doute, cette formulation paraîtra trop sommaire à Raffestin qui préfère - comme d'autres géographes, pas nécessairement majoritaires dans leur discipline - décrire la géographie humaine comme l'explicitation de tout ce que les êtres humains ont acquis de connaissance et de pratique de cette réalité qu'on nomme «espace» (ibid., p. 2). Il me semble néanmoins que l'attention prioritaire pour les modalités du lien ou pour les caractéristiques du lieu définissent deux orientations d'esprit différentes qu'il n'est d'ailleurs pas impossible de réunir et qu'il est éminemment souhaitable de réunir dans l'aide à la prise de déci$\operatorname{sion}^{18}$. Face à l'éventualité d'une fusion entre les cantons de Vaud et de Genève, notre collègue Jean-Bernard Racine fit observer les difficultés que soulève la réunion d'une entité urbaine - Genève - avec un canton «essentiellement rural historiquement». Il mit également l'accent sur les déséquilibres proprement vaudois entre le bassin lémanique «espace de flux qui s'homogénéise » et un arrièrepays «extrêmement cloisonné » qui pourrait se sentir marginalisé. L'argumentation présentée par Racine - y compris les réflexions sur l'hétérogénéité des institutions et des représentations mentales - fait preuve d'une sensibilité sociologique indéniable; mais celle-ci se double - pour le malheur de ses contradicteurs - d'un esprit géographique qui rend attentif aux «identités très fortes» des diverses entités de l'arrière-pays vaudois (Racine, 1997). On sent ici la réponse à l'exhortation d'un classique de la géographie humaine à «ouvrir les yeux et voir» (Brunhes, 1910, 1956, p. 282) mais aussi le reflet d'une conception sous-jacente qui rend explicite « how place and space are forces, and how they braid together nature and culture (which includes social relations and meaning) and help constitute the self» (Sack, 1997, p. 1; souligné par JCH).

Les orientations différentes mais complémentaires du sociologue et du géographe ne peuvent-elles pas être réunies en référence à la catégorie conceptuelle

17 Je traduis ici librement, en termes modernes, Dupréel qui, à ma connaissance, peut revendiquer la paternité de l'expression «esprit sociologique» (cf. Dupréel, 1948, pp. 379-381).

18 Qui semble concerner les géographes autant que les sociologues, si l'on en juge par l'idée de «bilan régional» mentionnée par Pierre George (1966, p. 19), par la tonalité générale des conclusions présentées par Raffestin et al. dans l'étude «Frontières et sociétés » (1975, pp. 197-198) ou encore par «the search for relevance in geographical research» signalée par Jeanne Wolfe (in Kobayashi et Mackenzie, 1989, p. 62). 
du «milieu »? C'est ce que suggèrent Jean Remy et Liliane Voyé lorsqu'ils présentent hypothétiquement la notion de milieu comme un concept analytique permettant de supprimer l'autonomie de «l'effet social» et de le relier au contraire «à d'autres niveaux d'explication» (1982, p. 35). L'idée est séduisante. Elle peut néanmoins susciter quelques malentendus inter- ou intradisciplinaires. Sur ce point, les sociologues cherchent généralement l'inspiration chez Durkheim. Celui-ci énonce en effet la règle selon laquelle «l'origine première de tout processus social de quelque importance doit être recherchée dans la constitution du milieu social interne» $(1895,1992$, p. 111). Cette notion de «milieu social interne» est constituée en analogie avec le «milieu interne des organismes». Il s'agit des «éléments de toute nature qui entrent dans la composition d'une société», de même que «l'ensemble des éléments anatomiques, avec la manière dont ils sont disposés dans l'espace, constitue le milieu interne des organismes » (ibid.). Pour Durkheim, les éléments qui composent ce «milieu social interne» sont de deux sortes: des choses et des personnes. Voilà qui semble offrir de larges perspectives à l'interdisciplinarité. Moins qu'on ne pourrait l'imaginer cependant car les «objets matériels » incorporés à la société sont ici «les produits de l'activité sociale antérieure, le droit constitué, les mœurs établies, les monuments littéraires, artistiques, etc.» Cette énumération est singulièrement plus restrictive que ce que pourrait souhaiter le géographe. Au surplus, ces éléments matériels du «milieu social interne» ne recèlent selon Durkheim «aucune puissance motrice» (ibid., p. 112). Dans ces conditions, il ne reste comme «facteur actif» que le milieu «proprement humain». Les caractéristiques pertinentes de celui-ci sont la «densité dynamique», c'est-à-dire «le nombre des individus qui sont effectivement en relations» (ibid.) et la «densité matérielle», à savoir «le nombre des habitants par unité de surface» pondéré par «le développement des voies de communication et de transmission» (ibid., p. 113). On est loin ici, on le voit, du «paradigme de l'écosystème » qu'invoquent Remy et Voyé en prenant appui sur les travaux antérieurs de l'Ecole de Chicago (1982, pp. 36 et ss). Ce n'est donc pas seulement le statut explicatif de la notion durkheimienne de «milieu social» qui doit être remis en question ${ }^{19}$ : c'est son extension et sa portée elles-mêmes. Il reste que son statut explicatif est également problématique. Dans De la division du travail social, quand Durkheim indique que «c'est donc dans certaines variations du milieu social qu'il faut aller chercher la cause qui explique les progrès de la division du travail» $(1893,1922$, p. 237), il semble bien qu'il ne s'agisse en l'occurrence que d'un raccourci analytique - qui s'impose sans doute dans le contexte d'argumentation qui est le sien - se substituant à un système d'interaction trop complexe pour pouvoir être décrit et analysé de façon plus précise en termes de variables. On peut en effet aller beaucoup plus avant dans la description des «effets de milieu». C'est ce que fait Mendras, par exemple, lorsqu'il décrit la société villageoise comme «un groupe d'inter-connaissance où chacun a une perception globale, et non fonctionnelle, de la personnalité d'autrui» car il y a constante «confusion des rôles» $(1967$, p. 322). L'expression «milieu social», couramment utilisée par les sociologues pour évoquer en termes généraux les

19 Par rapport auquel s'impose «une distance critique», selon les termes de Remy et Voyé (1982, p. 36). 
pressions diffuses ou les influences diverses que peut subir un individu, est fondée sur une analogie organique qu'on trouve également chez les classiques de la géographie. Ce n'est pourtant qu'à condition de définir très largement - beaucoup plus largement qu'on ne le fait d'habitude - «les produits de l'activité sociale antérieure » (Durkheim, 1895, 1992, p. 112) en y incluant les influences de très longue durée «sur le monde vivant» (Vidal de la Blache, 1922, p. 8) qu'elle peut se prêter aux espoirs de synthèse interdisciplinaire.

Quelle que soit néanmoins la conception qu'on se donne du milieu, l'espace cet espace que Sorre nous présentait comme consubstantiel à la géographie (1957, p. 99) - en est un des éléments clés. Le géographe localise et c'est ce qui le fait souvent confondre avec un cartographe. L'analyse sociologique exige également des localisations et le sociologue est ainsi enclin à traiter le géographe en aide de camp. Pourtant, la notion d'espace et la combinaison de raisonnements déductifs et inductifs qui intervient dans le passage de l'espace à la région (ibid., p. 32 et ss) créent des problèmes que ni la seule cartographie ni la pure spéculation intellectuelle ne peuvent résoudre. Il y a d'abord l'impossibilité pour la recherche géographique «d'enfermer les phénomènes qu'elle saisit dans un espace fini » qui serait la région (George, 1966, p. 9), celle-ci pouvant être tour à tour région naturelle, région géographique exprimant une synthèse entre «pseudo-déterminismes géographiques » et action humaine, région historique constituée malgré les conditions naturelles, enfin région économique répondant à certains impératifs de gestion (ibid.). Il y a ensuite les pièges que recèle l'idée de région naturelle ellemême. La Camargue, cet espace apparemment «naturel» et «déshérité », et pourtant «artificiel» et révélateur de «divers modèles idéologiques et techniques » (Mendras et Forsé, 1983, pp. 39-40) en fournit une illustration frappante. Un autre exemple, peut-être moins connu dans les régions de langue française, est celui des avatars de la bruyère du Jutland et des conceptions successives de l'espace naturel qui se sont affrontées à son sujet au Danemark (Sack, 1997, pp. 105107). Claude Raffestin résume toutes ces ambiguïtés en parlant des deux faces de l'espace, «l'une qui est le plan d'expression constitué par des surfaces, des distances et des propriétés et l'autre qui est le plan de contenu constitué par les surfaces, les distances et les propriétés aménagées qui sont signifiées par les acteurs » (1980, pp. 41-42). Pour établir plus clairement cette distinction entre l'espace comme donné et comme construit humain, il est tentant de faire usage du concept de «territorialité » emprunté à l'éthologie. Le territoire est «le produit des acteurs » et ceux-ci «produisent le territoire en partant de cette réalité première donnée qu'est l'espace» (ibid., p. 3) ${ }^{20}$. Et c'est cette distinction terminologique et conceptuelle qui a permis ultérieurement à Raffestin, s'adressant comme géographe à une audience de sociologues, de confirmer que «la géographie, à travers la territorialité, s'intéresse au lien social» (1989, p. 10). L'intérêt simultané pour le lien et pour le lieu étant ainsi précisé, il en résulte le recours à des termes communs à la géographie et à la sociologie: structures, maillages, réseaux, centralités, etc. $(1980 \text {, p. } 137)^{21}$. Recouvrent-ils les mêmes analogies pour le géographe et

20 Sur la charge idéologique que peut prendre la notion de territoire, la vision centralisatrice jacobine s'opposant à celle des vieux «pays », voir: Birnbaum, 1998.

21 Termes dont certains sont abondamment utilisés dans cette spécialité sociologique en voie d'autonomisation qu'est devenue l'analyse de réseaux (cf. par exemple Coenen-Huther, 1993). 
pour le sociologue? En d'autres termes, permettent-ils aux uns et aux autres de procéder par transposition et d'attribuer à l'objet étudié un «faisceau » de propriétés caractéristique d'un même objet «mieux connu ou tenu pour tel, pris pour point de repère ${ }^{22}$ ? Dans certains cas, on peut sans doute répondre sans trop d'hésitations par l'affirmative. Le terme de maillage, par exemple, évoque la dimension des ouvertures laissées entre les fils d'un filet de pêche. Pour le géographe, il peut exprimer d'abord «la projection d'un système de limites ou de frontières », ensuite «l'aire d'exercice des pouvoirs ou l'aire de capacité des pouvoirs » (Raffestin, 1980, p. 139). Voilà bien une analogie que le sociologue acceptera sans aucune difficulté. Il pourra se servir du terme dans les mêmes acceptions que le géographe. Mais il l'utilisera également dans un sens plus éloigné de la division du territoire. Quand il fera allusion par exemple à un système de protection sociale ou à des réseaux de solidarité «au maillage plus ou moins serré»-cette expression est devenue courante -, il ne fera plus, ou plus nécessairement, référence à un découpage territorial mais à un tissu social abstrait. Dans ce sens métaphorique, un «maillage serré» signifiera un mécanisme d'entraide ne laissant pour compte que très peu d'individus: rares sont ceux qui risqueront de passer «entre les mailles du filet». Il en va de même en matière d'exclusion ou de désaffiliation sociale. Quand Robert Castel évoque des «zones de vulnérabilité» ou seules «des frontières fragiles » séparent la masse du peuple de «sa frange désaffiliée » (1995, p. 108), il fait un usage métaphorique de ces références spatiales bien que le caractère analogique des termes soit partiellement voilé par l'évocation de la figure du vagabond d'Ancien Régime, précurseur du désaffilié social d'aujourd'hui. C'est d'ailleurs, notamment, la prise de conscience de cet aspect métaphorique qui pousse Castel à préférer le terme «désaffiliation» au terme «exclusion», mettant ainsi en doute - pour reprendre les termes de Chazel - «le processus de valeur ajoutée au plan de la connaissance» qui résulterait d'un transfert analogique trop large (1990, pp. 211-212) ${ }^{23}$. Pour utiliser avec rigueur la notion d'exclusion, explique Castel, «il faudrait qu'elle corresponde à des situations caractérisées par une localisation géographique précise» liée à l'existence d'une subculture cohérente et à une base ethnique (ibid., p. 15; souligné par JCH) ${ }^{24}$.

Ainsi, on le voit, les sociologues qui ont recours à des concepts spatiaux peuvent évoquer des réalités très proches de celles qui préoccupent les géographes. C'est principalement le cas des sociologues qui - ruralistes ou spécialistes du milieu urbain - privilégient tout autant qu'eux le territoire ${ }^{25}$. La spatialité des

22 Je m'appuie ici sur le bel article de François Chazel, L'analogie et ses limites (1990, p. 184) où l'auteur emprunte à Jean-Blaise Grize l'idée de «faisceau d'aspects », inséparable de la démarche analogique.

23 A la différence d'Alain Touraine qui juge au contraire indispensable « de parler d'exclusion et de lutte contre l'exclusion, en dépit de la pertinence des critiques de Castel» (1999, pp. 56-57). Comme le dirait Chazel, il n'existe pas, dans nos disciplines, «de limites bien établies à l'emploi des analogies, ni donc de voie royale clairement tracée» (1990, pp. 210-211).

24 Ce qui lui semble être le cas dans les ghettos américains mais pas - ou pas encore - en France, pays sur lequel porte principalement son analyse, en dépit du phénomène «beur» (Castel, 1995, p. 15).

25 On notera à ce propos que Pierre George a pu écrire successivement un livre intitulé Sociologie et géographie (1966) et un chapitre intitulé Sociologie géographique dans le Traité de sociologie publié sous la direction de Georges Gurvitch (1967). 
sociologues rejoint également celle des géographes quand les phénomènes observés révèlent une interférence nette de la dimension sociale et de la dimension spatiale, par exemple dans l'examen des relations entre mobilité sociale et mobilité spatiale (Coenen-Huther, 1990). Pour le sociologue, il s'agit pourtant le plus souvent d'une spatialité purement figurative. Tout sociologue a en tête une visualisation plus ou moins précise et plus ou moins consciente de la société qui fait l'objet de son attention. Cette image, pour adopter les termes de Dupréel (1948), repris ultérieurement par Henri Janne (1960), est celle d'une «structure en quadrillé » où «des figures oblongues, verticales et parallèles» représentent des domaines d'activité tandis que «d'autres figures allongées, sortes de bandes ou de rubans traversant horizontalement les premières » représentent des classes ou des niveaux de statuts (Dupréel, 1948, p. 125). Cette façon de conceptualiser un espace social sans lien nécessaire avec un support géographique quelconque a engendré d'autres notions reposant sur une analogie spatiale évidente: les «coordonnées sociales» définissant la position d'un individu dans une structure, la « distance sociale» qui s'établit entre des individus ayant des «positions sociales » éloignées l'une de l'autre, éventuellement à la suite d'un processus de «mobilité sociale» individuelle ou collective, ascendante ou descendante. Comme d'autres concepts visant à situer des faits ou des processus à l'aide de coordonnées espace-temps, ces notions constituent l'héritage de la physique sociale du XVII ${ }^{e}$ siècle (Sorokin, 1928, 1938, Chap. I). Les analogies qu'elles proposent prennent tout leur sens par rapport à un modèle mécaniste du système social qui précéda le modèle organiciste (Buckley, 1967, pp. 8-11) mais qui n'en reste pas moins présent dans la conceptualisation sociologique actuelle. Dans la sociologie contemporaine, la notion de «champ» qui joue un rôle crucial dans l'œuvre théorique de Pierre Bourdieu relève également d'un appareil conceptuel physicospatial. Le «champ» est un «espace social» qui constitue à la fois un «champ de forces» s'imposant aux individus et un «champ de luttes» au sein duquel les individus s'affrontent en fonction de leur position dans la structure du champ de forces (Bourdieu, 1994, pp. 54-55). Les divers «champs» sont des sphères d'activité plus ou moins autonomes selon les cas, et peuvent entretenir des relations-frontières les uns avec les autres à la manière des sous-systèmes de Parsons ${ }^{26}$. Bien que l'espace social comme «champ» soit a priori tout aussi déconnecté de l'espace du géographe que la «structure en quadrillé » de Dupréel, il ne s'en prête pas moins à une visualisation procédant à la fois d'une logique de stratification et d'une logique fonctionnelle comme en témoigne la schématisation de l'espace des positions sociales et de l'espace des styles de vie (Bourdieu, 1979, pp.140-141 $)^{27}$. Bien que la préoccupation géographique de localisation soit ici absente, le sociologue qui conçoit l'espace social comme le lieu de relations de pouvoir rejoint à cet égard le géographe qui entend organiser sa réflexion -

26 S'agissant du «champ artistique», il est d'ailleurs intéressant de constater que la visualisation qu'en offre Bourdieu se prête, tout comme les systèmes et sous-systèmes de Parsons, à des emboîtages successifs (1994, p. 74).

27 L'axe vertical de stratification présente une hiérarchisation en termes de volume de «capital global» alors que l'axe horizontal fonctionnel met en évidence le poids respectif du «capital» spécifique à chaque sphère d'activité (qu'on pourrait aussi bien convenir d'appeler «segment fonctionnel»). 
et rénover la géographie politique - à l'aide du «fil-guide» du pouvoir (Raffestin, 1980 , p. 1). A l'heure actuelle, la seule représentation visualisée de la société qui puisse prétendre faire concurrence à la visualisation classique par croisement d'axes verticaux et d'axes horizontaux est celle du centre et de la périphérie, le centre étant occupé par les personnes dont les positions sont les plus stables et des zones délimitées par des cercles concentriques successifs suggérant des situations de vulnérabilité de plus en plus grave ${ }^{28}$. Dans ce cas, l'analogie s'établit avec les catégories conceptuelles de la géopolitique, identifiant par exemple des «zones de dégradation» (Sorre, 1957, p. 95) qui pourraient correspondre aux «zones de vulnérabilité» de l'analyste de l'espace social moderne.

Faisant usage de la notion d'espace - qu'ils se réfèrent à un espace réel ou à un espace conceptuel - géographes et sociologues se retrouvent aux prises avec des problèmes semblables. Il s'agit en effet, pour les uns et pour les autres, de circonscrire l'espace considéré. A part certains cas d'insularité peu fréquents, écrit Pierre George, «il n'y a jamais d'espace fini à l'égard de tous les phénomènes », ce qui implique nécessairement la superposition d'espaces finis différents (1966, p. 9). La limite de phénomènes complexes - commodément recouverts par la notion de milieu - en devient donc floue et ne s'établit que par contrastes. Ainsi en est-il du phénomène urbain et de ce qui a pu être observé - par exemple par Simmel - sur le milieu de la grande ville. La spécificité du fait urbain n'apparaît que dans la relation entre l'urbain et le rural (ibid., p. 14). Pour reprendre les termes de Boudon, on pourrait dire que le géographe, comme le sociologue, se trouve placé devant le problème méthodologique de la transformation de systèmes indéfinis en systèmes définis, c'est-à-dire de systèmes constitués par des ensembles de composantes aisément repérables et dont le nombre est défini (1968, pp. 99-102). Le sociologue cherche son salut dans la construction de types idéaux et dans la dichotomisation; le géographe dans la superposition de cartographies partielles qui produisent en fin de compte des noyaux centraux et des zones intermédiaires. A ce sujet, Raffestin fait observer que «la limite est... une classe générale, un ensemble dont la frontière est un sous-ensemble» (1980, p. 150). Guillaume De Greef, un sociologue bruxellois du tournant du siècle, un peu oublié de nos jours ${ }^{29}$, avait autrefois défendu l'idée que les formes de sociabilité et d'organisation propres à un "agrégat social », c'est-à-dire, dans ses termes, à la combinaison d'une population et d'un territoire (1908a, p. 264), dépendent notamment de la nature des frontières avec l'extérieur (1908b, pp. 9 et ss). De Greef pose en effet en principe que «l'organisation interne d'une société [est] en corrélation avec sa structure générale notamment vis-à-vis de l'extérieur» (1908b, p. 20; souligné par JCH). Ceci l'amène à conclure que la «frontière-limite extérieure de toute société fait partie de la structure de celle-ci» (ibid., p. 25). Cette conclusion est importante car elle permet d'envisager les rapports d'un peuple à son territoire en s'affranchissant de notions déterministes périmées. C'est ici

28 Notre collègue Volker Bornschier, de l'Université de Zurich, fait usage d'une modélisation de ce type (cf. Bornschier et Keller, 1994, pp. 83-112; en particulier Die Schichtung nach Statusgruppen im Zentrum-Peripherie-Modell, p. 86).

29 A son sujet, voir mon article sur La sociologie de Tönnies, les formes de sociabilité et l'Ecole sociologique de Bruxelles (1997a, pp. 93-94) sur lequel je m’appuie ici. 
l'historien qui intervient dans les débats qui concernent la sociologie et la géographie. L'histoire de Russie en fournit un bon exemple. En deçà et au-delà de la révolution russe, on peut discerner un «fait générateur» au sens tocquevillien: le rapport à la frontière. Un Etat longtemps faible, incertain de ses frontières, menacé de l'extérieur pendant des siècles, apparaît sans cesse tenté par une politique d'extension territoriale dont le principe est de mettre la plus grande distance possible entre un envahisseur potentiel et le centre du pouvoir ${ }^{30}$. Ainsi, le mythe de la mentalité russe façonnée par la relation à un espace démesuré, exprimé sur le plan littéraire par Gogol (Les Âmes mortes, 1842) ou par Tchekhov (La Steppe, 1888), est remplacé par une interprétation d'ordre institutionnel.

Qu'il s'agisse d'observer des faits ou des catégories de faits dans un cadre spatial déterminé, ou encore d'appliquer la méthode comparative ${ }^{31}$, la référence à la totalité s'impose. Elle s'impose, au plan de la morphologie sociale, lorsqu'on souhaite réaliser un inventaire «domicilié dans l'espace» (George, 1967, p. 255). Elle s'impose tout autant lorsqu'on désire comparer «des familles de faits, des formes de groupement et de corrélations» (ibid.). Quels seront alors les «domiciles de base » qui donneront leur sens aux comparaisons? L'intention généralisante prend ici des formes différentes pour la géographie et pour la sociologie bien qu'on puisse se fonder dans les deux cas sur le concept de «société globale». La géographie, note Pierre George, se situe sur un plan horizontal, «son fini est l'espace occupé par un type de société globale ou un ensemble de rapports sociaux » (1966, p. 16). Cette perspective ne peut qu'être accentuée par le volet physique de la recherche géographique qui pousse à l'extension maximale. Ceci apparaît très clairement chez Brunhes qui écrit «il n'y a pas sur l'écorce terrestre de compartiment fermé; il peut y avoir des cloisons, il n'y a pas de clôtures » $(1910,1956$, p. 11). Et de citer sur ce point son maître Vidal de la Blache qui observe: «aucune partie de la terre ne porte en elle seule son explication. Bien plus, le jeu des conditions locales ne se découvre avec quelque clarté qu'autant que l'observation s'élève au-dessus d'elles et qu'on est en mesure d'embrasser les analogies que ramène naturellement la généralité des lois terrestres ${ }^{32}$. La sociologie au contraire, observe Pierre George, se situe sur un plan vertical, «son fini est la totalité sociale, la société globale» (1966, p. 16). On perçoit ici la référence implicite à la notion maussienne de «fait social total » qui pourrait être le pendant d'un «fait géographique total» esquissé par Brunhes et Vidal de la Blache. Pour Mauss, on s'en souvient, les faits sociaux sont «totaux» ou «généraux» dans la mesure où ils mettent en cause «la totalité de la société et de ses institutions» ou «un très grand nombre d'institutions » (1923-24, éd. 1968, p. 274). L'expression «société globale», telle qu'elle est couramment utilisée par les sociologues, comporte néanmoins une ambiguïté fondamentale. Elle suggère en effet une intention totalisante, englobant non seulement les différents niveaux micro-, méso- et macrosociaux de la réalité sociale, mais également les différentes sphères d'activité. Dès lors, elle invite à l'universalisation du discours en laissant dans le vague les limites réelles de l'entité sociale dont il est question. En fait, la plupart des analyses sociologiques à visée générale portent sur des sociétés définies et limitées

\footnotetext{
J'ai développé ceci plus en détail dans mon Tocqueville (1997b, pp. 109-111).

Les deux démarches que Pierre George assigne à une «sociologie géographique» (1967. p. 255).

Paul Vidal de la Blache, cité par Brunhes (1910, 1956, pp. 11-12).
} 
par des frontières politiques ou sur la comparaison de telles sociétés. Ceci signifie que depuis le XIX $\mathrm{X}^{\mathrm{e}}$ siècle jusqu'à nos jours, la sociologie s'est développée principalement dans le cadre de l'Etat, c'est-à-dire, le plus souvent, de l'Etat-nation. Il ne s'agit pas - ou pas nécessairement - d'une sociologie de l'Etat, au sens où Raffestin parle d'une géographie politique s'identifiant sous l'influence ratzelienne à une géographie de l'Etat (1980, p. 17), mais bien d'une sociologie dont les modes de pensée sont largement sous l'influence du cadre étatique. Pour les géographes comme pour les sociologues - mais probablement bien davantage que la plupart des sociologues ne l'imaginent - le problème de méthode récurrent reste l'articulation entre le «plan horizontal» du géographe et le «plan vertical» du sociologue. A cet égard, les travaux portant sur la région transfrontalière franco-genevoise (Raffestin et al., 1975) l'indiquent assez clairement, l'exploration résolue de l'axe horizontal ne peut que réintégrer la dimension historique dans les procédés d'investigation courants de la recherche sociologique.

A la suite de Pierre George (1966, pp. 4-5), Denise Pumain et Jean-Bernard Racine nous font observer que l'homo geographicus ne s'identifie pas entièrement à «l'homme habitant» de Maurice Le Lannou (Pumain et Racine, 1999, p. 79). Et de fait, certains procédés de conceptualisation axés sur la notion de réseau plutôt que sur celle de lieu semblent actuellement séduire les géographes, surtout lorsqu'ils ont à traiter avec des décideurs préoccupés d'aménagement du territoire (Gaudin et Pumain, 2000). Ceci ne pourrait qu'être accueilli favorablement par les sociologues si cette orientation ne véhiculait de nouvelles métaphores biologiques dont ils ont appris à se méfier et qu'ils ne peuvent que percevoir comme une régression; c'est le cas, par exemple, de l'analogie fondée sur le système neuronal (ibid). Il n'en reste pas moins que l'habitation semble bien occuper une «place exceptionnelle» dans «la hiérarchie géographique des faits humains » (Brunhes, 1910, 1956, pp. 21-22). Sans doute, cette place était plus centrale encore lorsque l'attention du géographe se portait sur des sociétés plus stables où la continuité de l'habitat pouvait être considérée comme «le témoignage et le symbole de la durée d'une civilisation » (Sorre, 1957, pp. 54-56). Mais l'idée de stabilité relative, voire de sécurité, tout comme d'ailleurs un sentiment de responsabilité, restent liés à la notion de domicile (Sack, 1997, p. 14). Comme j'ai déjà eu l'occasion de l'indiquer ailleurs, l'existence d'un domicile inviolable est devenue un des traits de notre culture tout comme un des fondements de l'Etat de droit (Coenen-Huther, 1995, p. 103). Plus généralement, pour l'enfant, le monde s'identifie tout d'abord avec le milieu familial. A un certain stade de développement, toutes les personnes de référence (significant others) appartiennent à ce micro-monde associé au lieu où l'on habite qui devient ainsi - pour rester sur le mode métaphorique - le «port d'attache» d'où l'individu «entame son voyage à travers la vie» (Berger et Berger, 1972, p. 77). Et ce point fixe garde une charge affective que l'on retrouve dans la résonnance particulière que prennent des expressions comme «à la maison», «thuis», «zu Hause», «at home», «doma», etc. ${ }^{33}$ Cette dernière perspective est héritée à la fois de la psycho-sociologie de George Herbert Mead et de la sociologie phénoménologique d'Alfred Schütz. L'homo sociologicus n'est donc pas ce Heimatloser de partout et de nulle part qui

33 Sack en fait mention pour ce qui concerne les connotations et les implications étymologiques du terme «home» dans la langue anglaise (1997, p. 15). 
ne serait pas concerné par les ancrages géographiques. Peut-être, après tout, sociologues et géographes ont-ils une maison commune...

\section{Département de Sociologie \\ Université de Genève}

\section{RÉFÉRENCES BIBLIOGRAPHIQUES}

Berger, Peter L. et Berger, Brigitte, Sociology. A biographical Approach, New York, Basic Books, 1972.

Berthelot, Jean-Michel, La construction de la sociologie, Paris, Presses Universitaires de France, Coll. «Que sais-je?», N 2602, 1991.

Birnbaum, Pierre, La France imaginée. Déclin des rêves unitaires?, Paris, Fayard, 1998.

Bornschier, Volker et Keller, Felix, Die Statusgruppenschichtung als Ursache von Konflikt und Devianz, in «Schweizerische Zeitschrift für Soziologie/Revue suisse de sociologie», Vol. 20, $\mathrm{N}^{\circ} 1,1994$, pp. 1-13.

Boudon, Raymond, L'analyse mathématique des faits sociaux, Paris, Plon, 1967.

Boudon, Raymond, A quoi sert la notion de structure? Paris, Gallimard, 1968.

Boudon, Raymond, L'idéologie. L'origine des idées reçues, Paris, Fayard, 1986.

Bourdieu, Pierre, La distinction. Critique sociale du jugement de goût, Paris, Minuit, 1979

Bourdieu, Pierre, Raisons pratiques. Sur la théorie de l'action, Paris, Seuil, Points/Essais, 1994.

Brunhes, Jean, La géographie humaine, 1910. Paris, Presses Universitaires de France. Edition abrégée, 1942, $3^{\text {e } e ́ d ., ~} 1956$.

Brunhes, Jean et Vallaux, Camille, La géographie de l' histoire, Paris, Alcan, 1921.

Buckley, Walter, Sociology and Modern Systems Theory, Englewood Cliffs, N.J., Prentice-Hall, 1967.

Busino, Giovanni, La permanence du passé. Questions d' histoire de la sociologie et d'épistémologie sociologique, Genève, Droz, 1986.

Castel, Robert, Les métamorphoses de la question sociale. Une chronique du salariat, Paris, Fayard, 1995.

Chazel, François, L'analogie et ses limites, in: G. Duprat, Connaissance du politique, Paris, Presses Universitaires de France, Coll. «Questions», 1990, pp. 181-213.

Chazel, François, Pouvoir, in: R. Boudon, Traité de sociologie, Paris, Presses Universitaires de France, 1992, pp.195-226.

Coenen-Huther, Jacques, Sociologie et interdisciplinarité: modalités, problèmes, perspectives, in «Schweizerische Zeitschrift für Soziologie/Revue suisse de sociologie», Vol. 15, N 1, 1989, pp. 1-13.

Coenen-Huther, Jacques, Relations d'amitié, mobilité sociale et mobilité spatiale, in «Espaces et Sociétés », Paris, $\mathrm{N}^{\mathrm{os}}$ 54-55, 1990, pp. 51-65.

Coenen-Huther, Jacques, Analyse de réseaux et sociologie générale, in «Flux, Cahiers scientifiques internationaux Réseaux et Territoires », Paris, La Documentation française, $\mathrm{N}^{\circ} 13 / 14$, juilletdécembre 1993, pp. 33-40.

Coenen-Huther, Jacques, Observation participante et théorie sociologique, Paris, L'Harmattan, Coll. «Logiques sociales», 1995.

Coenen-Huther, Jacques, La sociologie de Tönnies, les formes de sociabilité et l' Ecole sociologique de Bruxelles, in «Recherches sociologiques», Louvain-la-Neuve, Vol. XXVIII, Nº 1, 1997a, pp. 91 96.

Coenen-Huther, Jacques, Tocqueville, Paris, Presses Universitaires de France, Coll. «Que sais-je?», $\mathrm{N}^{\mathrm{o}} 3213,1997 \mathrm{~b}$. 
Crozier, Michel et Friedberg, Erhard, L'acteur et le système. Les contraintes de l'action collective, Paris, Seuil, 1977.

De Greef, Guillaume, La structure générale des sociétés, Tome I, La loi de limitation, Bruxelles, Larcier, et Paris, Alcan, 1908a.

De Greef, Guillaume, La structure générale des sociétés, Tome II, Théorie des frontières et des classes, Bruxelles, Larcier, et Paris, Alcan, 1908b.

Doorn, J.A.A. Van, Beeld en betekenis van de Nederlandse sociologie [Image et importance de la sociologie néerlandaise], Utrecht, Bijleveld, 1964.

Dupréel, Eugène, Sociologie générale, Paris, Presses Universitaires de France, 1948.

Durkheim, Emile, De la division du travail social, Paris, Alcan, 1893, 4éd., 1922.

Durkheim, Emile, Les règles de la méthode sociologique, Paris, Alcan, 1895, réédition, PUF, 1937, réimpression, Coll. «Quadrige», 1992.

Gaudin, Jean-Pierre et Pumain, Denise, Réseaux de villes, réseaux urbains, réseaux de pouvoir, in «Revue Européenne des Sciences Sociales», Tome XXXVIII, 2000, nº 117.

George, Pierre, Sociologie et géographie, Paris, Presses Universitaires de France, 1966.

George, Pierre, Sociologie géographique, in: G. Gurvitch, Traité de sociologie, Tome I, Paris, Presses Universitaires de France, $3^{\mathrm{e}}$ éd. revue et mise à jour, 1967, pp. 255-274.

Gourou, Pierre, Pour une géographie humaine, Paris, Flammarion, 1973.

Gurvitch, Georges, La vocation actuelle de la sociologie, Tome I: Vers la sociologie différentielle, Paris, Presses Universitaires de France, 1950, $3^{\mathrm{e}}$ éd. revue, 1963.

Janne, Henri, Un modèle théorique du phénomène révolutionnaire?, in «Annales, Economies, Sociétés, Civilisations », Vol 15, Nº 6, pp. 1138-1154.

Kobayashi, Audrey et Mackenzie, Suzanne, Remaking Human Geography, Boston, Unwin Hyman, 1989.

Martindale, Don, The Nature and Types of Sociological Theory, Second Edition, New York, Harper and Row, 1981.

Mauss, Marcel, Essai sur le don, 1923-24, in «Sociologie et anthropologie», Paris, Presses Universitaires de France, 1968.

Mendras, Henri, Sociologie du milieu rural, in: G. Gurvitch, Traité de sociologie, Tome I, Paris, Presses Universitaires de France, $3^{\mathrm{e}}$ éd. revue et mise à jour, 1967, pp. 315-331.

Mendras, Henri et Forsé, Michel, Le changement social. Tendances et paradigmes, Paris, Armand Colin, 1983.

Mucchielli, Laurent, La découverte du social. Naissance de la sociologie en France, Paris, La Découverte, 1998.

Piaget, Jean, Le système de la classification des sciences, in: J. Piaget, éd., Logique et connaissance scientifique, Paris, Gallimard, La Pléiade, 1967, pp. 1151-1224.

Pumain, Denise et Racine, Jean-Bernard, L'Homo Geographicus : de l'emprunt à l' empreinte, « Revue Européenne des Sciences Sociales », Tome XXXVII, No 113, 1999, pp. 77-86.

Racine, Jean-Bernard, La fusion éclair Vaud-Genève de Philippe Pidoux? Alors ça, non! Interview recueillie par Michel Perrin, in «Tribune de Genève», 20 novembre 1997.

Raffestin, Claude, Guichonnet, Paul et Hussy, Jocelyne, Frontières et Sociétés. Le cas franco-genevois, L'Age d'Homme, Lausanne, 1975.

Raffestin, Claude, Pour une géographie du pouvoir, Paris, Librairies techniques, 1980.

Raffestin, Claude, Allocution du Vice-Doyen de la Faculté des Sciences Economiques et Sociales de l'Université de Genève, in: Association Internationale des Sociologues de Langue Française, Actes du XIII ${ }^{\mathrm{e}}$ Colloque (Genève, 29 août-2 septembre 1988), Le lien social. Identités personnelles et solidarités collectives dans le monde contemporain, Tome I, pp. 10-11, Université de Genève, 1989.

Raffestin, Claude, La Suisse et l'immigration: pour un accueil actif, in «Schweizerische Zeitschrift für Soziologie/Revue suisse de sociologie », Vol. 18, N 3, 1992, pp. 695-706. 
Reclus, Elisée, Nouvelle géographie universelle. La terre et les hommes, Tome I, Paris, Hachette, 1875.

Remy, Jean et Voyé, Liliane, Milieu, rapport social et conflit, in: J. Remy, éd., Milieu et rapport social, AISLF, Comité de recherche «Politiques locales », Institut de Sociologie, Université de Bruxelles, 1980, pp. 35-71.

Sack, Robert David, Homo Geographicus : A Framework for Action, Awareness, and Moral Concern, Baltimore, Johns Hopkins University Press, 1997.

Sorokin, Pitirim, Contemporary Sociological Theories, New York, Harper and Row, 1928. Tr. fr. Les théories sociologiques contemporaines, Paris, Payot, Bibliothèque scientifique, 1938.

Sorre, Maximilien, Rencontres de la géographie et de la sociologie, Paris, Marcel Rivière, Petite bibliothèque sociologique internationale, 1957.

Touraine, Alain, Comment sortir du libéralisme?, Paris, Fayard, 1999.

Valade, Bernard, Introduction aux sciences sociales, Paris, Presses Universitaires de France, Coll. «Premier cycle», 1996.

Vervoort, C. E., De sociologie-opleiding in Nederland [L'enseignement de la sociologie aux PaysBas], in: I. Rademaker, Ed., Sociologie in Nederland [La sociologie aux Pays-Bas], Deventer, Van Loghum Slaterus, 1979, pp. 98-123.

Vidal de la Blache, Paul, Principes de géographie humaine, Paris, Armand Colin, 1922. 